\title{
Resistencia De Materiales-Ingeniería Mecánica Estudio De Caso: Degradación Del Acero Astm A36 Sometido Al Fenómeno De Termofluencia
}

\author{
Jorge Isaías Caicedo Reyes \\ Javier Enrique Orna Chávez \\ Otto Fernando Balseca Sampedro \\ Diego Fernando Mayorga Pérez \\ Edwin Fernando Viteri Nuñez.
}

Docente- Investigador de la Facultad de Mecánica

Escuela Superior Politécnica de Chimborazo - Ecuador

Catalina Margarita Verdugo Bernal

Docente- Investigador de la Facultad de Recursos Naturales

Escuela Superior Politécnica de Chimborazo - Ecuador

\begin{abstract}
Creep is the simultaneous combination of load and high temperature for a time period in a material, and in which mechanical behavior is a new field of research. In this paper, the results of the experimental study are discussed in order to obtain information on the effect of the microstructural level, which resulted in the degradation of ASTM A36 steel affect of moderate effort $\left(1223.14 \mathrm{Kg} / \mathrm{cm}^{2}\right)$ in a temperature range of 500 to $650{ }^{\circ} \mathrm{C}$. The methodology is analytical, qualitative, quantitative, deductive logic and experimental. The tests were conducted according to ASTM E139 using flat specimens which section is reduced and sized according to ISO 6892: 1998 (E). In the tested specimens, samples were extracted from the fractured zone in order to conduct metallographic and micro hardness tests. The results showed that at the temperature of $500^{\circ} \mathrm{C}$ in a maximum of 3-day test, the material did not show any significant microstructural level transformations, but from $550^{\circ} \mathrm{C}$ to $650{ }^{\circ} \mathrm{C}$ it clearly showed the effects of creep in the material microstructural level, both by the presence of cavity characteristics, which are due to the movement of dislocations, like the level of hardness by the formation of soft phases.
\end{abstract}

Keywords: Thermo-fluency, dislocation, degradation, steel ASTM A36 


\section{Resumen}

Termofluencia es la combinación simultánea de carga y temperatura elevada a la que se expone un material por un periodo de tiempo y en el cual su comportamiento mecánico constituye un nuevo campo de investigación. En este trabajo se exponen los resultados del estudio experimental con el objetivo de obtener información sobre el efecto a nivel microestructural que determinó la degradación del acero ASTM A36 por efecto del esfuerzo moderado aplicado $\left(1223.14 \mathrm{Kg} / \mathrm{cm}^{2}\right)$ en un rango de temperatura de 500 a $650^{\circ} \mathrm{C}$. $\mathrm{La}$ metodología empleada es analítica, cualitativa, cuantitativa, lógica deductiva y experimental. Los ensayos se efectuaron según la norma ASTM E139 utilizando los especímenes planos de sección reducida dimensionados según la norma ISO 6892:1998 (E). A los especímenes ensayados se les extrajeron probetas de la zona fracturada para realizarles ensayos metalográficos y de microdureza. Los resultados mostraron que a la temperatura de $500^{\circ} \mathrm{C}$ en un tiempo máximo de 3 días de ensayo, el material no presenta transformaciones relevantes a nivel microestructural, pero a partir de $550^{\circ} \mathrm{C}$ se evidenciaron claramente los efectos de termofluencia en el material tanto a nivel microestructural por la presencia de cavidades características debido al movimiento de dislocaciones, como a nivel de su dureza por la formación de fases blandas.

Palabras claves: Termofluencia, dislocación, degradación, acero ASTM A36

\section{Introducción}

La termofluencia es un fenómeno que lo definen como aquel que se produce por la combinación de carga y temperatura elevada, considerando como elevada a una T>0.3-0.4T fusión. (Ali Morovat, y otros, 2012, págs. 786788). El ensayo de termofluencia proporciona información primordial sobre la correlación entre las tensiones que actúan sobre el material, la temperatura y el tiempo de falla.

También se enuncia, que la termofluencia, es un fenómeno poco familiar (si se lo quiere relacionar con las deformaciones elásticas o plásticas) el cual depende de la temperatura a la que se encuentra trabajando el material, y se dice que la temperatura a la que este empieza a producirse será cuando

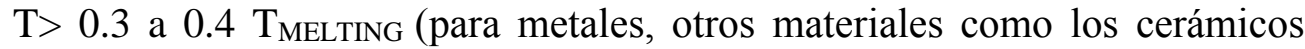
poseen su propio rango de temperatura). (Ashby \& D., 2011, págs. 311-312) 
Gráfico No.01. Curva ideal de termofluencia

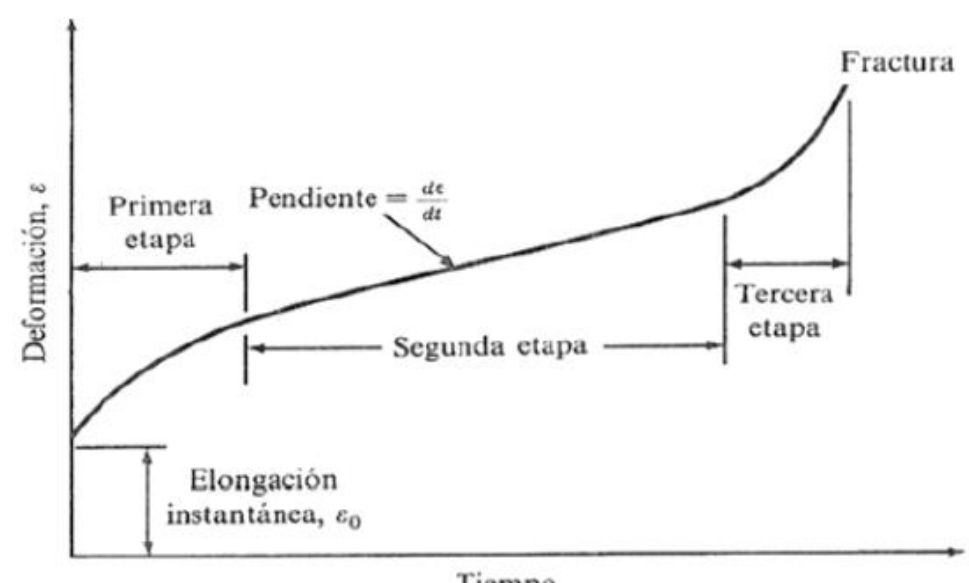

Tiempo

Fuente: (Caicedo, 2014, pág. 16)

La curva ideal de termofluencia (Deformación-Tiempo) se muestra en el Gráfico No.01, la deformación instantánea que se observa es resultado del esfuerzo aplicado la cual consta de tres etapas, una primera etapa en la que los mecanismos de deformación endurecen el material, por lo que la velocidad de deformación decrece y con ello la tangente de la curva; luego se tiene una segunda etapa en la que la pendiente de la curva permanece constante ya que los mecanismos de endurecimiento por deformación se equilibran con los mecanismos de aniquilación de endurecimiento por deformación, con la particularidad que además aparecen los mecanismos generadores de dislocaciones y los mecanismos de aniquilación de dislocaciones. Luego tenemos una tercera etapa donde los mecanismos de aniquilación de endurecimiento por deformación y los mecanismos generadores de dislocaciones prevalecen de tal manera que el material se deforma rápidamente y las dislocaciones producidas en su interior conllevan rápidamente a la fractura del material. (Gonzales, 2003, págs. 231-236)

A lo largo de una extensa revisión de la literatura sobre ésta temática, se nota que éste efecto es poco estudiado en la actualidad, existiendo una escasez de datos experimentales sobre la fluencia de aceros estructurales, lo que quiere decir que no existen estudios que hagan referencia el efecto a nivel microestructural en el acero ASTM A36, por lo que, no se incluyen reglas de prevención para este fenómeno conocido como termofluencia en las normas de construcción. Algunos estudios sobre termofluencia, en otros materiales, se describen a continuación:

En ciertas fuentes literarias se ha investigado acerca de los métodos por los cuales es posible estimar o predecir la vida de un material, refiriéndonos específicamente a los aceros ferríticos, sometidos a 
termofluencia, para este fin se han analizado ciertos procedimientos ya existentes para posteriormente emplear el más eficiente, siendo éste la máquina de vectores de soporte para regresión, ya que indica que éste es un método menos errado comparado por ejemplo con el método de las redes neuronales. Es importante destacar que éste tipo de estimaciones provienen de modelos matemáticos que, aunque son pequeños, aún tienen cierto porcentaje de error. En el desarrollo de ésta investigación no se ha tratado los efectos de éste problema a nivel estructural. (Díaz, Valencia Morales, \& Pérez. E., 2009, págs. 53-58).

En cuanto al efecto de la termofluencia en aceros, como por ejemplo el inoxidable austenítico 316, ciertos estudios han empleado el ensayo mediante identación a distintas temperaturas $\left(650,675\right.$ y $700{ }^{\circ} \mathrm{C}$ ) para posteriormente caracterizar su comportamiento mediante la velocidad de termofluencia, el esfuerzo y tiempo de ruptura. En esta investigación se muestran fotomicrografías posteriores al ensayo donde se observan los efectos a nivel microestructural de la termofluencia para este acero en específico. (Saucedo-Muñoz, Komazaki, Hashida, \& \& López-Hirata, 2015, págs. 51(19)).

(Williams- leir, 1983, págs. 73-78), también ha dedicado como fin de su investigación el determinar "expresiones analíticas" que permitan predecir el tiempo de vida de un acero estructural reforzado con concreto y expuesto a altas temperaturas provenientes de fuego. Determinando finalmente, que la exposición a altas temperaturas si provocan un deterioro más rápido en el material, como ya se conoce.

En estudios como el de (V. K. R. Kodur, 2010, págs. 1327-1341), se destaca la relevancia de analizar los efectos de la temperatura en elementos cargados y como la fluencia afectará el rendimiento de dicho acero. Se brinda como ejemplo el caso típico de un miembro estructural actuando bajo un incendio y se investiga como el fuego reduce el tiempo de vida de estos elementos empleando modelos matemáticos y un software especializado como ANSYS. Finalmente se culmina la investigación indicando que, de no considerarse este efecto en el diseño, éste no será completamente conservador (su factor de seguridad se reduce).

Debido a la limitada información sobre la resistencia de aceros a la termofluencia, (V. K. Kodur, 2015, págs. 1-10) se ve la necesidad de estudiar el efecto de la fluencia a altas temperaturas específicamente en las deformaciones que se puedan presentar. Se llevaron a cabo ensayos en el material especificado y a las temperaturas comunes a las que se someterían en caso de incendio, dando como resultado que, en efecto la temperatura tiene una influencia considerable en la deformación progresiva que suceda durante la inflamación. 
Así también, existen escasos estudios referentes a este tema en específico para aceros estructurales de similar composición química al acero ASTM A36, por ejemplo, (Harmathy \& Stanzak, 1970, págs. 1-23) evalúan las propiedades de tensión y fluencia a elevadas temperaturas de dos aceros estructurales y un acero pretensado que son ampliamente utilizados en la industria de la construcción.

(Ali Morovat, y otros, 2012, págs. 786-793); han realizado una comparación entre los modelos teóricos de Harmathy y Fields con resultados experimentales de termofluencia en un acero ASTM A992 utilizando dos tipos de perfiles, concluyendo que, estos tienen un gran margen de error comparado con los datos reales obtenidos, señalando así la necesidad de modelos teóricos más precisos.

(Cowan, 2014, págs. 1-9) su documento se enfoca en la modelación matemática de la fluencia a alta temperatura mediante el ensayo del acero ASTM A992, en la que propone una relación manejando una función de seno hiperbólico.

(Zeng, Tan, \& Huang, 2003, págs. 951-970), en su investigación proponen un método semi-analítico para predecir la resistencia al fuego de columnas de acero, a través de un modelo matemático simplificado, concluyendo que éste último exhibe un margen de error pequeño, admisible para el diseño de estructuras.

El acero ASTM A36 es muy comercial por su maleabilidad, soldadura, corte, precio, etc., llegando así a ser muy utilizado por el gremio industrial y artesanal en la fabricación de muchos equipos, instalaciones y componentes mecánicos, tales como: estructuras metálicas, calderos, intercambiadores de calor, hornos, etc. Se sabe que las propiedades del material están vinculadas con la composición química del mismo, su procesamiento y la microestructura resultante; las propiedades que dependen de la microestructura se llaman "structure sensitive properties", por ejemplo, el límite elástico o la dureza (Brnic, Canadija, Turkalj, \& Lanc, 2010, págs. 1083-1089); por lo tanto se concluye, que es necesario realizar un estudio minucioso del comportamiento de este material frente al fenómeno de termofluencia.

Éste análisis, como se puede notar, es de relevancia ya que éste fenómeno probablemente involucre degradación microestructural que al ignorarla (como en la actualidad) puede o podría conllevar a serios errores de diseño, convirtiéndose éstos en un riesgo potencial para las vidas humanas, como en el caso de estructuras cerradas donde existe una alta probabilidad de producirse un incendio que simule las condiciones para que ocurra termofluencia. En este trabajo se busca evaluar el comportamiento de este material frente al fenómeno de termofluencia, por ello se realizó un análisis de su microestructura resultante y dureza, con el fin de contribuir así con datos experimentales que sean considerados en el diseño y que garanticen una 
operación segura y confiable de los equipos en cuyos componentes se haga uso del acero ASTM A36.

\section{Metodología y procedimiento experimental}

En la presente investigación, para llevar a cabo la caracterización microestructural del material sometido a termofluencia se ha utilizado los métodos: analítico, cualitativo, cuantitativo, experimental y lógico deductivo. (La investigación científica o método experimental constituye un método empírico enfocado al estudio directo del fenómeno, con el fin de determinar sus propiedades y relaciones útiles a la investigación a través del control de las condiciones en que se desarrolla. El método lógico deductivo aplica los principios descubiertos a casos particulares a partir de un enlace de juicios, para descubrir consecuencias desconocidas de principios conocidos.) Se han realizado distintos ensayos de termofluencia en el acero ASTM A36 a distintas temperaturas de interés: $500,550,600$ y $650^{\circ} \mathrm{C}$, siguiendo el procedimiento indicado en la norma ASTM E139; en el ensayo el parámetro de estudio es la deformación plástica producida por una carga que actúa durante prolongados periodos de tiempo, seguido de la preparación de las diferentes muestras metalográficas y la medición de microdureza.

\section{- $\quad$ Equipo y especímenes utilizados}

Los ensayos de termofluencia se realizaron en la máquina construida en un programa de maestría (Gráfico No.02.).

Gráfico No.02. Equipo empleado en el ensayo de termofluencia.

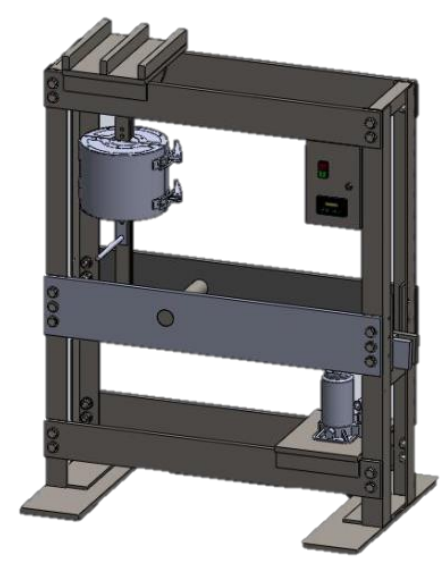

Fuente: (Caicedo Reyes, 2014, p. 58)

Consiste en una estructura en la que se sustenta un eje que sirve de pivote para una palanca; la fuerza para tensionar los especímenes es aplicada con una gata hidráulica en el extremo de mayor longitud de la palanca con respecto al eje (pivote), la gata hidráulica dispone un manómetro con la finalidad de poder apreciar la carga aplicada. Por medio de un mecanismo 
acoplado a la palanca se tensiona los especímenes. La temperatura requerida se consigue con un horno tipo libro de resistencias eléctricas, en cuyo interior se censa la temperatura con una termocupla tipo $\mathrm{K}$ que opera con un controlador digital tipo K. (Caicedo Reyes, 2014, p. 34)

Para realizar los ensayos de termofluencia a las temperaturas consideradas se emplearon especímenes planos de sección reducida obtenidos en sentido paralelo a la laminación de la plancha y dimensionados según la norma ISO 6892:1998 (E) (Gráfico No.03).

Gráfico No.03. Geometría y dimensiones del espécimen empleado

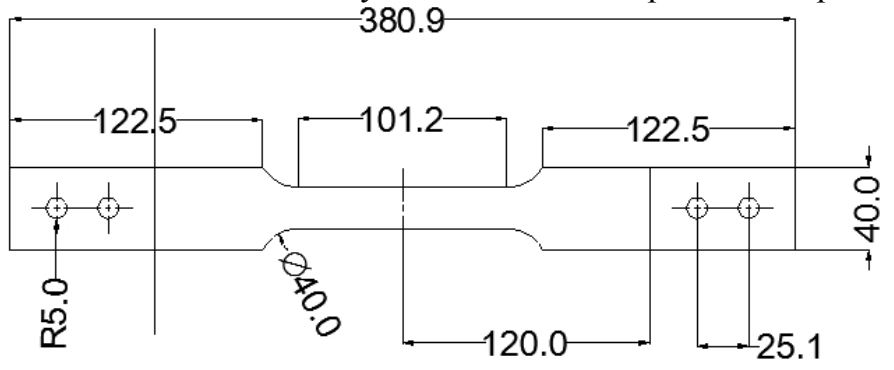

Fuente: (Caicedo, 2014, pág. 60)

\section{- Determinación del Esfuerzo a aplicar}

Con el fin de determinar un esfuerzo adecuado para que se produzca termofluencia a las temperaturas consideradas, se realizaron ensayos de tracción, encontrando el comportamiento del material mediante los diagramas Esfuerzo-Deformación (Gráfico No.04.), de donde se obtuvo el esfuerzo máximo correspondiente a cada temperatura.

Gráfico No.04. Diagramas Esfuerzo-Deformación a las temperaturas de 500, 550, 600 y 650 ${ }^{\circ} \mathrm{C}$.

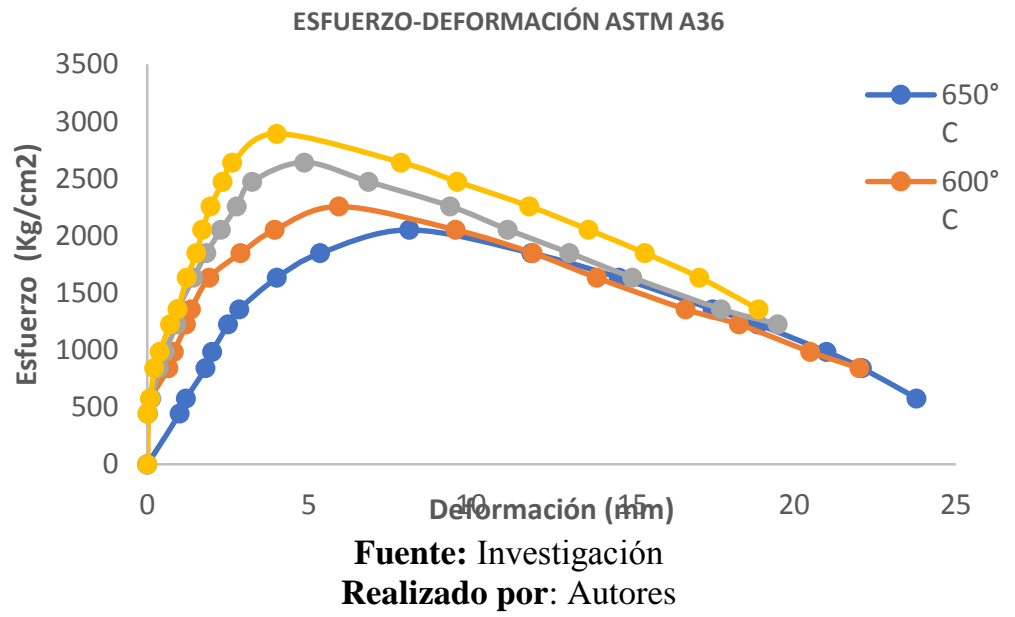

Los datos de nuestro interés del Grafico No.4, los registramos en el Cuadro 1 para una mejor apreciación cuantitativa, se observa que a mayor 
temperatura disminuye el esfuerzo que soporta el acero ASTM A36, pero la deformación aumenta conforme se incrementa la temperatura aplicada en cada ensayo.

Cuadro 1: Registro de esfuerzo máximo y deformación a las temperaturas de 500, 550, 600 y $650^{\circ} \mathrm{C}$.

\begin{tabular}{|c|c|c|}
\hline $\begin{array}{c}\text { Temperatura } \\
\left({ }^{\circ} \mathrm{C}\right)\end{array}$ & $\begin{array}{c}\text { Deformación } \\
(\mathrm{mm})\end{array}$ & $\begin{array}{c}\text { Esfuerzo Máximo } \\
(\mathrm{kg} / \mathrm{cm} 2)\end{array}$ \\
\hline 500 & 4,00 & 2892 \\
\hline 550 & 4,85 & 2640 \\
\hline 600 & 5,93 & 2256 \\
\hline 650 & 8,10 & 2052 \\
\hline
\end{tabular}

Fuente: Investigación

Realizado por: Autores

El parámetro de Larson-Miller es el más utilizado para representar los datos de fluencia-esfuerzo de ruptura para aleaciones resistentes a la termofluencia a elevadas temperaturas (Smith, 2006, págs. 7-36). Con los datos anteriores y utilizando el parámetro de Larson-Miller se extrapoló los datos encontrados, considerando como tiempo máximo de ensayo 3 días; determinando así el esfuerzo de $1223 \mathrm{~kg} / \mathrm{cm}^{2}(119,95 \mathrm{MPa})$ correspondiente al 47,98\% del esfuerzo de fluencia del acero ASTM A36 (250 MPa), por lo tanto, se consideró válido este esfuerzo debido a que siempre que la temperatura es relativamente alta, y se aplica una carga por debajo del nivel del esfuerzo máximo, la deformación plástica que ocurre es dependiente del tiempo, lo que se conoce como termofluencia. (Ashby \& Jones, 2008, págs. 311-322)

\section{- Descripción de los ensayos}

Definidos los parámetros de temperatura y esfuerzo, se procedió a realizar 3 ensayos por cada temperatura de interés, manteniendo el esfuerzo constante para todos los ensayos. El procedimiento para efectuar el ensayo de termofluencia menciona que, hay que llegar a la temperatura de interés y una vez estabilizada esta temperatura hay que aplicar rápidamente la carga.

De los especímenes sometidos a termofluencia, se extrajeron probetas de la vecindad de la fractura para efectuarles ensayos metalográficos a 100 micrones y de microdureza a $100 \mathrm{um}, 2,4,6,8,10,13$ y $18 \mathrm{~mm}$ a partir de la fractura, con la finalidad de analizar cambios a nivel microestructural y de su dureza por efecto del fenómeno de termofluencia

\section{Resultados}

A $500^{\circ} \mathrm{C}$ la curva de termofluencia (Gráfico No.05) se encuentra incompleta con respecto a la curva ideal, ya que el fenómeno sólo alcanza su segunda etapa sin llegar a producir la fractura en el tiempo máximo considerado de ensayo (3 días). Al obtener estos resultados es simple darse 
cuenta que el fenómeno de termofluencia no produjo efectos en el material; además el Cuadro 3 muestra que el porcentaje de reducción de área y de elongación son relativamente bajos.

Gráfico No.05. Gráfica Deformación-Tiempo. Temperatura $500^{\circ} \mathrm{C}$.

ASTM A36 TERMOFLUENCIA $500^{\circ} \mathrm{C}$

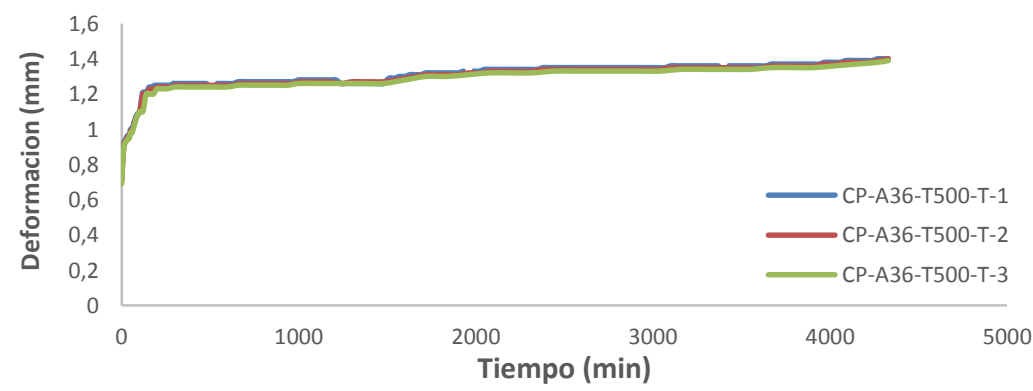

Fuente: Investigación

Realizado por: Autores

En los Gráficos No. 06, 07 y 08 resulta claramente apreciable las tres etapas de la curva de termofluencia, llegando a producirse la fractura del material en el tiempo indicado en el Cuadro 3 para cada temperatura de interés. Nótese que, a mayor temperatura los porcentajes de reducción de área y de elongación aumentan drásticamente pese a que los tiempos de ensayo disminuyeron, es así que a la temperatura de $650^{\circ} \mathrm{C}$ se obtiene un porcentaje de reducción promedio del área de $91,47 \%$ y una elongación promedio de $47,6 \%$ en un tiempo de ensayo promedio de 29 minutos.

Gráfico No.06. Gráfica Deformación-Tiempo. Temperatura $550^{\circ} \mathrm{C}$.

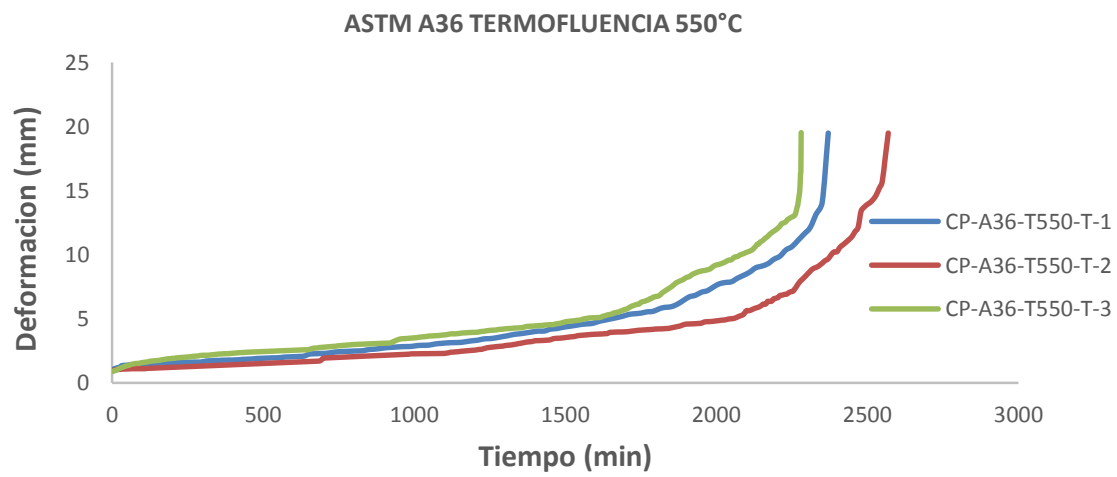

Fuente: Investigación

Realizado por: Autores 
Gráfico No.07. Gráfica Deformación-Tiempo. Temperatura $600^{\circ} \mathrm{C}$.

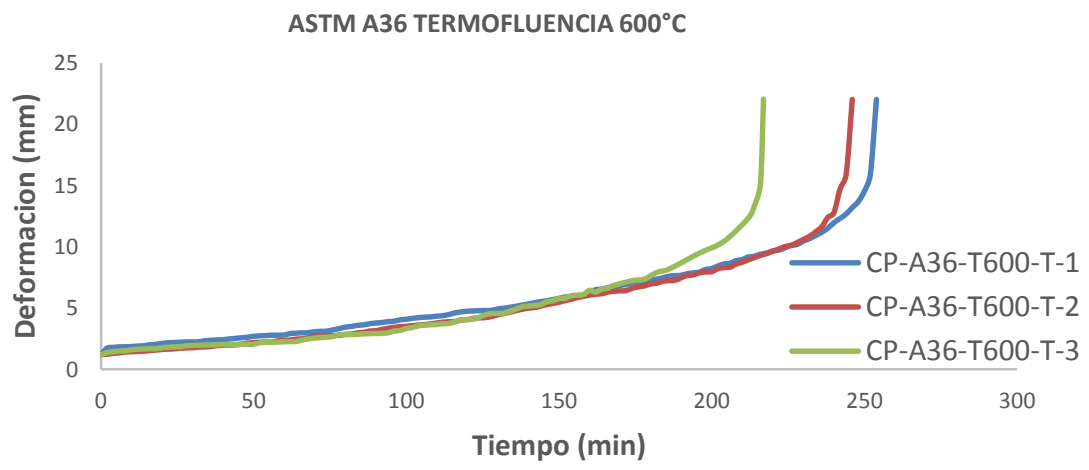

Fuente: Investigación

Realizado por: Autores

Gráfico No.08. Gráfica Deformación-Tiempo. Temperatura $650^{\circ} \mathrm{C}$.

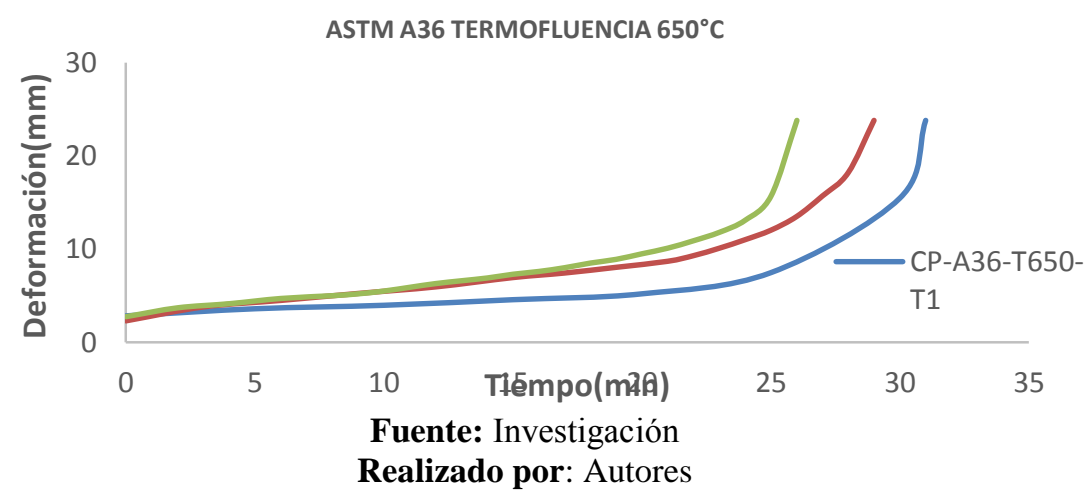

La deformación instantánea que presentaron los ensayos de termofluencia lo muestra el Cuadro 2.

Cuadro 2: Deformación instantánea a las temperaturas de $500,550,600$ y $650{ }^{\circ} \mathrm{C}$.

\begin{tabular}{|c|c|c|c|c|}
\hline Temperatura $\left({ }^{\circ} \mathrm{C}\right)$ & 500 & 550 & 600 & 650 \\
\hline $\begin{array}{c}\text { Deformación } \\
\text { instantánea }(\mathrm{mm})\end{array}$ & 0,70 & 0,90 & 1,20 & 2,50 \\
\hline
\end{tabular}

\footnotetext{
Fuente: Investigación

Realizado por: Autores
}

Nótese que, cada vez es mayor la deformación instantánea a medida que la temperatura del ensayo aumenta.

Se observa que, a medida que la temperatura del ensayo aumenta, el tiempo de ensayo disminuye. Además se cuantificó el porcentaje de reducción de área y de elongación que sufrió el material, mostrando los resultados en el Cuadro 3. 
Cuadro 3: Duración de ensayo y porcentaje de reducción de área y elongación a las temperaturas de $500,550,600$ y $650{ }^{\circ} \mathrm{C}$.

\begin{tabular}{|c|c|c|c|}
\hline $\begin{array}{c}\text { Ensayo } \\
{ }^{\circ} \mathrm{C}\end{array}$ & $\begin{array}{c}\text { Duración Ensayo } \\
(\mathrm{min})\end{array}$ & $\begin{array}{c}\text { Reducción Área } \\
(\%)\end{array}$ & $\begin{array}{c}\text { Elongación } \\
(\%)\end{array}$ \\
\hline 500 & 4320 & 4,93 & 2,820 \\
\hline 550 & 2503 & 82,84 & 39,00 \\
\hline 600 & 239 & 87,73 & 44,06 \\
\hline 650 & 29 & 91,47 & 47,60 \\
\hline
\end{tabular}

Fuente: Investigación

Realizado por: Autores

A continuación, el Gráfico No.09 muestra que a medida que la temperatura de ensayo aumenta, la pendiente de la curva DeformaciónTiempo aumenta drásticamente a partir de $550^{\circ} \mathrm{C}$.

Gráfico No.09. Incremento de la pendiente de la curva Deformación-Tiempo a medida que la temperatura de ensayo aumenta.

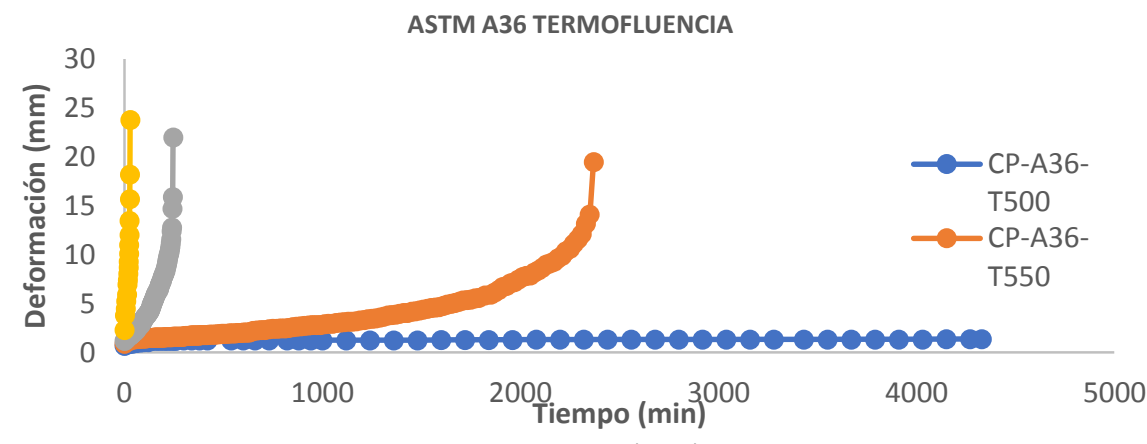

Fuente: Investigación

Realizado por: Autores

Esto da indicios de que a la temperatura de $500^{\circ} \mathrm{C}$ y carga de $1223 . \mathrm{kg} / \mathrm{cm}^{2}(119,95 \mathrm{MPa})$ el acero ASTM A36 no presenta degradación por termofluencia, pero sí a las temperaturas de 550,600 y $650^{\circ} \mathrm{C}$. Para consolidar lo mencionado se efectuó un análisis metalográfico a las probetas extraídas de la zona de la fractura.

\section{- $\quad$ Ensayos Metalográficos}

A 100 micrones de la fractura se efectuó el ensayo metalográfico a $400 x$, las probetas se atacaron con Nital al $4 \%$ y las fotografías que se muestran en los Gráficos No 08, 09, 10 y 11 corresponden a los especímenes ensayados a las temperaturas de $500,550,600$ y $650^{\circ} \mathrm{C}$ respectivamente. Cabe mencionar que debido a las temperaturas a las que se realizaron los ensayos predominaron los mecanismos de deslizamiento de dislocaciones sobre los mecanismos de difusión, produciendo el movimiento de las cavidades a través de los límites de grano (Gonzales, 2003, págs. 232-233). 
Al realizar el análisis metalográfico en las probetas extraídas de los especímenes ensayados a $500^{\circ} \mathrm{C}$ (Gráfico No. 10) se puede observar que no hubo un cambio notable con respecto a su microestructura a temperatura ambiente, tampoco presenta las fallas granulares como cavidades $\mathrm{R} o \mathrm{~W}$ que son características de este fenómeno, por lo que se confirma que no existió termofluencia.

Gráfico No.10. Microestructura. Temperatura de ensayo $500^{\circ} \mathrm{C}$ a $100 \mu \mathrm{m}$ de la fractura.

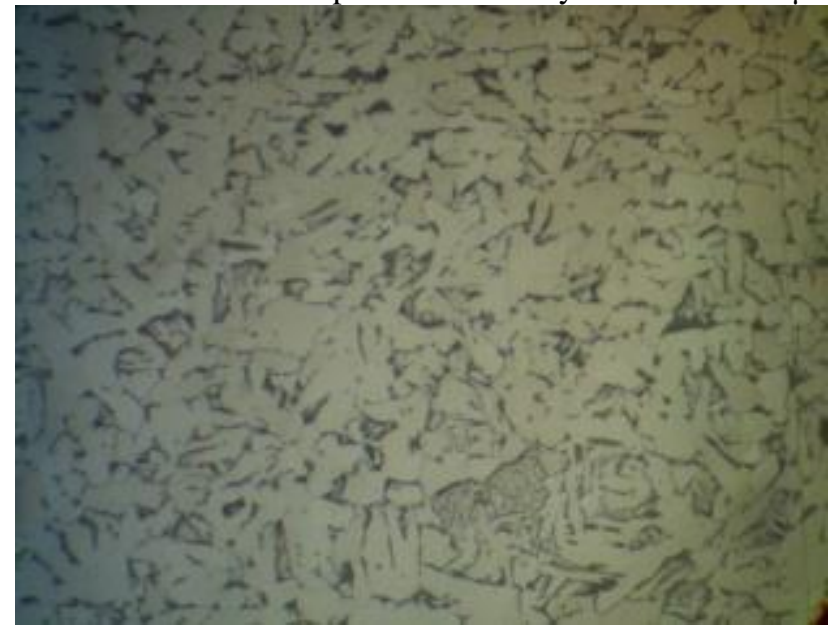

\section{Fuente: Investigación \\ Realizado por: Autores}

En la micrografía del Gráfico No.11, del espécimen ensayado a $550^{\circ} \mathrm{C}$ se observa la aparición de cavidades tipo $\mathrm{R}$ a lo largo de los límites de grano debido, éstas son cavidades esféricas y muestran que el material comenzó a ser afectado por el fenómeno de termofluencia. Aunque son de pequeño tamaño, constituyen un sumidero de concentración de esfuerzos que promueven la falla del material.

Gráfico No.11. Microestructura. Temperatura de ensayo $550^{\circ} \mathrm{C}$ a $100 \mu \mathrm{m}$ de la fractura.

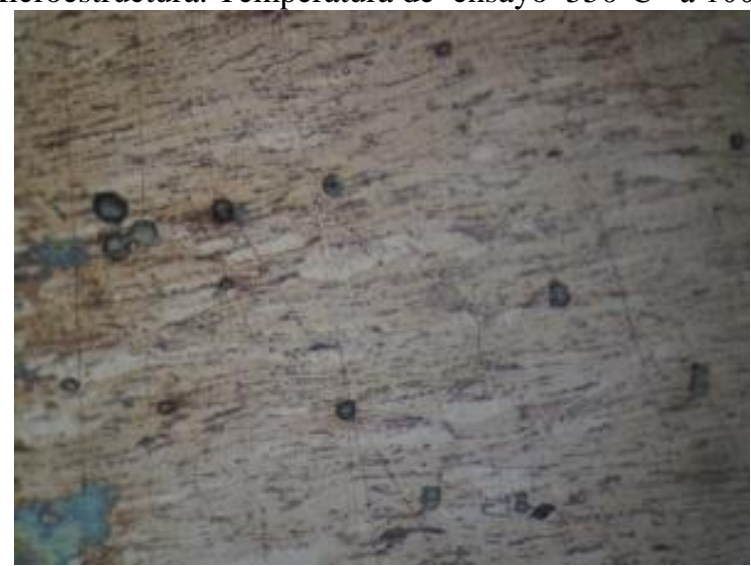

Fuente: Investigación

Realizado por: Autores 
Las micrografías de las probetas extraídas de los especímenes ensayados a 600 y $650^{\circ} \mathrm{C}$ (Gráficos No. 12 y 13) respectivamente, presentan un tipo diferente de cavidades, éstas se denominan tipo $\mathrm{W}$ y son producto del desgarre en las uniones triples de los granos, para su aparición se requiere de mayor energía (temperatura), son de mayor tamaño ya que las cavidades tipo $\mathrm{R}$ con mayor energía se desplazan fácilmente por los límites de grano y se agrupan con las cavidades tipo $\mathrm{W}$, como resultado, las cavidades son de mayor tamaño y geometría irregular alargada en la dirección de la carga aplicada. Por su forma y tamaño estas cavidades son mucho más críticas que las anteriores y provocan que el material se fracture en menor tiempo, como se verifica en el Cuadro 3.

Gráfico No.12. Microestructura. Temperatura de ensayo $600^{\circ} \mathrm{C}$ a $100 \mu \mathrm{m}$ de la fractura.

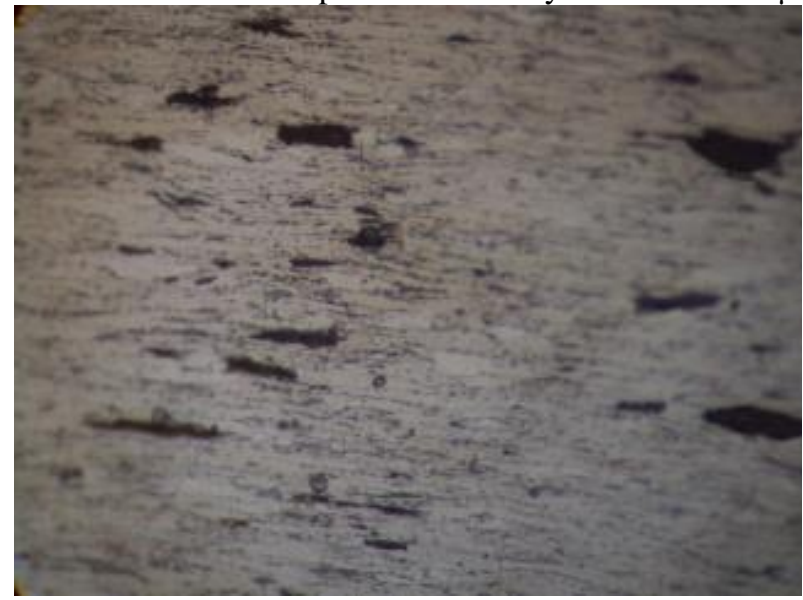

Fuente: Investigación

Realizado por: Autores

Gráfico No.13. Microestructura. Temperatura de ensayo $650^{\circ} \mathrm{C}$ a $100 \mu \mathrm{m}$ de la fractura.

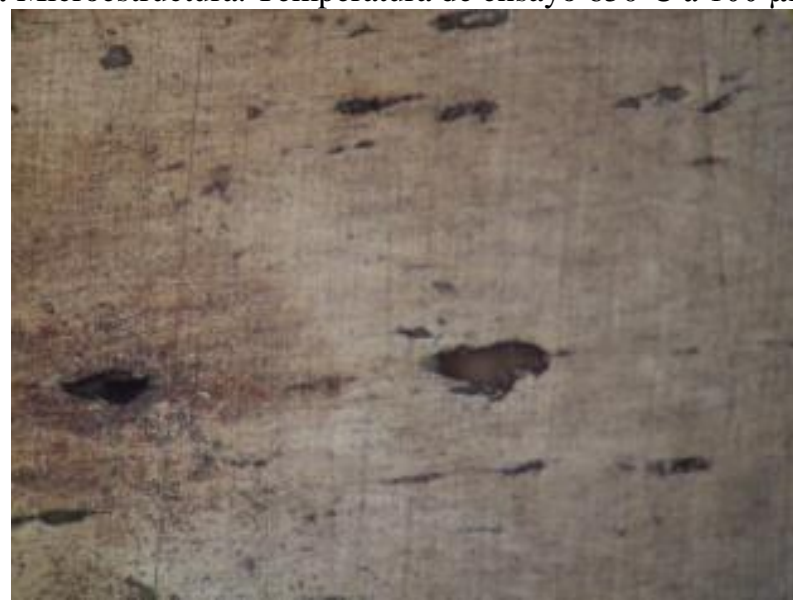

Fuente: Investigación

Realizado por: Autores 
En todas las micrografías anteriores, excepto en la de $500^{\circ} \mathrm{C}$ se observa como los granos se han alargado en el sentido de aplicación de la carga, debido también en parte a los mecanismos de fluencia por difusión (Gonzales, 2003, págs. 232-233)

Lo expuesto muestra que a partir de $550^{\circ} \mathrm{C}$ con carga moderada de $1223.14 \mathrm{~kg} / \mathrm{cm}^{2}(119,19 \mathrm{MPa})$ la termofluencia degrada considerablemente el acero ASTM A36 a nivel microestructural; para consolidar aún más este hecho, se realizaron ensayos de microdureza para determinar la influencia de la degradación a nivel microestructural sobre la dureza del material.

\section{- $\quad$ Ensayos de Microdureza}

Se realizó un barrido a partir de la fractura y a las distancias de: 100 um, 2, 4, 6, 8, 10,13 y $18 \mathrm{~mm}$ se realizaron ensayos de microdureza ya que en ésta zona se apreciaban cambios microestructurales, los resultados promedios se muestran a continuación en el Gráfico No.14.

Gráfico No.14. Gráfica de Microdureza-Distancia a partir de la fractura de las muestras extraídas de los especímenes ensayados a $500,550,600$ y $650^{\circ} \mathrm{C}$.

MICRODUREZA-DISTANCIA - ASTM A36

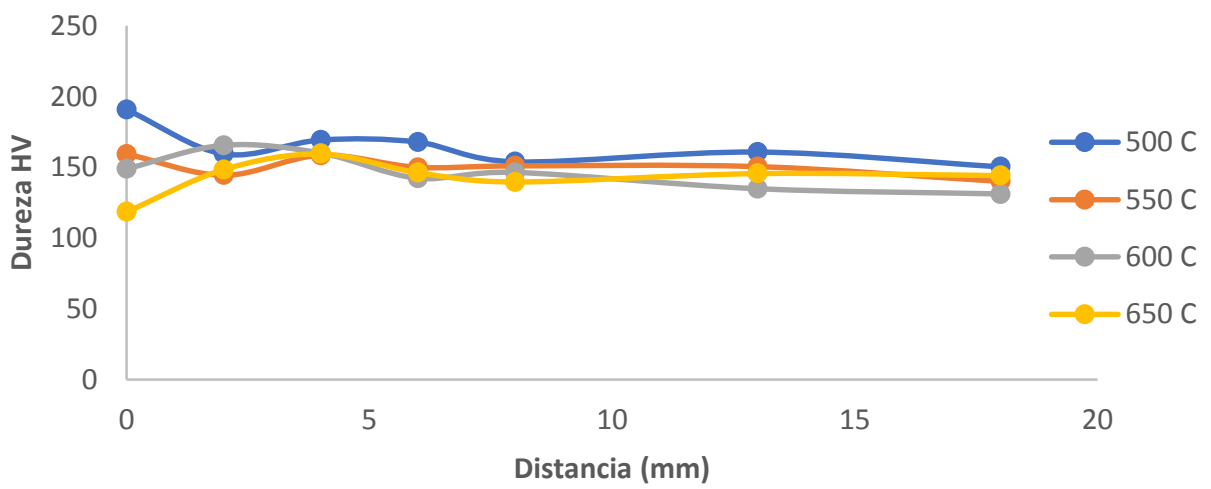

Fuente: Investigación

Realizado por: Autores

En el Gráfico No.14 se observa que en la zona más cercana a la fractura (100 um) se produce una variación de dureza. A la temperatura de $500^{\circ} \mathrm{C}$ el material presenta un endurecimiento por deformación y éste es el comportamiento habitual del acero ASTM A36 a temperatura ambiente, pero a partir de $550^{\circ} \mathrm{C}$ observamos que al incrementarse la temperatura de ensayo el material se torna más blando, pues predominan los mecanismos que dan lugar al aparecimiento de fases blandas, que hacen que el material no se endurezca por deformación, esto muestra que a nivel microestructural se dio un proceso competitivo entre los mecanismos de endurecimiento por 
deformación y los mecanismos de formación de fases blandas, predominando éstos últimos.

En los ensayos de termofluencia realizados se determinó que a partir de $550^{\circ} \mathrm{C}$ el material se vio afectado a nivel microestructural, evidenciándose además que a nivel de su propiedad física (dureza) también se produjo una afectación por dicho fenómeno.

\section{Conclusiones}

Luego del ensayo a $500^{\circ} \mathrm{C}$, la microestructura obtenida es muy similar a la del material en estado de suministro, por lo que se evidencia no haber sufrido afectación alguna por termofluencia, ya que no presenta degradación microestructural (cavidades) y su comportamiento en la curva DeformaciónTiempo presenta únicamente dos de las tres etapas de termofluencia sin llegar a la fractura; y aún tiene la capacidad de endurecerse por deformación, así lo muestra el ensayo de dureza realizado en la cercanía de la fractura, permitiendo concluir que éste material puede trabajar normalmente hasta una temperatura de $500^{\circ} \mathrm{C}$ y a un esfuerzo que no sobrepase los $1223 . \mathrm{kg} / \mathrm{cm}^{2}$ (119,95 MPa).

La termofluencia está caracterizada por la presencia de cavidades en la microestructura del material, apareciendo en un inicio cavidades tipo $\mathrm{R}$ (pequeñas y de forma circular) a lo largo de los límites de grano, y de tipo W en los puntos de unión triple de los granos (de mayor tamaño y geometría irregular), estas cavidades se convierten en sumideros de esfuerzos, que llevan a la fractura del material en un menor tiempo conforme aumenta la temperatura.

Los mecanismos de termofluencia que degradan al material, son los producidos por el movimiento de las dislocaciones; la velocidad con que se mueven las dislocaciones por los bordes de los granos, se ve favorecida con el incremento de la temperatura y por un grano de menor tamaño, permitiendo que se agrupen y formen cavidades de mayor tamaño rápidamente. Así también contribuyen a esta degradación, aunque en menor porcentaje los mecanismos de difusión impulsados por la energía que produce la tensión aplicada, éstos se encargan de la deformación de los granos en la dirección de la aplicación de la carga, produciendo granos irregulares que hacen que la fractura del material sea más propensa.

La dureza en la cercanía de la fractura de las distintas probetas ensayadas, muestra una relación inversa con la temperatura de ensayo, esto se debe al proceso competitivo entre los mecanismos de endurecimiento por deformación y los mecanismos de formación de fases blandas, predominando éstos últimos al incrementar la temperatura, lo que indica que a nivel físico el material también resulta afectado por termofluencia. 


\section{References:}

1. Ali Morovat, M., Lee, J. W., Engelhardt, M. D., Taleff, E. M., Helwig, T. A., \& Segrest, V. A. (2012). Creep properties of ASTM A992 steel at elevated temperature. Elseiver Journal, 786-793.

2. Ashby, M. F., \& Jones, D. R. (2008). Materiales para ingenieria, vol. 1, Introducción a las propiedades, las aplicaciones y el diseño. En M. F. Ashby, \& D. R. Jones, Materiales para ingenieria, vol. 1, Introducción a las propiedades, las aplicaciones y el diseño. Barcelona: Reverté.

3. Ashby, M., \& D., J. (2011). Engineering Materials: An introduction to properties applications and design. En A. MF, \& J. DRH, Engineering Materials: An introduction to properties applications and design. (págs. 311-312). Oxford: Elsevier.

4. Brnic, J., Canadija, M., Turkalj, G., \& Lanc, D. (2010). Structural steel ASTM A709-behavior at uniaxial tests conducted at lowered and elevated temperatures, short-time creep response, and fracture toughness calculation. . Journal of engineering mechanics, 1083-1089.

5. Caicedo, I. (2014). Estudio del efecto de termofluencia sobre el hierro fundido gris perlítico como material alternativo y su incidencia en la degradación microestructural en espejos de la cámara de combustión de los hornos industriales para pan. 44--65: Universidad Técnica de Ambato.

6. Cowan, M. (2014). Modeling of high temperature creep in ASTM A992 structural steels. Journal Homepage, 1-9.

7. Díaz, D., Valencia Morales, C. A., \& Pérez. E., \&. M. (2009). Modelo de máquinas de vectores de soporte para regresión aplicado a la estimación de la tensión de ruptura por termofluencia en aceros ferríticos. . Revista Facultad de Ingeniería Universidad de Antioquia, 53-58.

8. Gonzales, J. L. (2003). Metalurgia Mecánica. En J. L. Gonzales, Metalurgia Mecánica (págs. 232-233,745). Mexico: Limusa.

9. Harmathy, T. Z., \& Stanzak, W. W. (1970). Elevated Temperature Tensile and Creep Properties of Some Structural and Prestressing Steels. Journal Homepage, 1-23.

10. Loor, J., \& Morales, F. (2015). Ensayo de termofluencia del acero AISI-SAE 1018 y construcción del modelo predictivo de vida. Guayaquil: Escuela Superior Politécnica del Litoral.

11. Saucedo-Muñoz, M. L., Komazaki, S. I., Hashida, T., \& \& LópezHirata, V. M. (2015). Ensayo de termofluencia de indentación en un acero inoxidable austenítico 316. . Revista de Metalurgia, 51(1). 
12. Smith, W. (2006). Fundamentos de la ciencia e ingeniería de materiales. En W. Smith, Fundamentos de la ciencia e ingeniería de materiales (págs. Capítulo 7, página 36). Mexico: McGrawHill.

13. V. K. Kodur, E. M. (2015). Effect of temperature on creep in ASTM A572 in ASTM A572 high-strenght low-alloy steels. Materials and Structures, 1-10.

14. V. K. R. Kodur, M. M. (2010). Effect of high temperature creep on the fire response of restrained steel beams. Materials and structures, 43(10), 1327-1341.

15. Williams- leir, G. (1983). Creep of structural steel in fire: analytical expressions. Fire and Materials. Wiley, 73-78.

16. Zeng, J., Tan, K., \& Huang, F. (2003). Primary creep buibkling of steel columns in fire. Journal of Constructional Steel Research, 951-970. 